\title{
Stretching and break-up of saliva filaments during speech: A route for pathogen aerosolization and its potential mitigation
}

\author{
M. Abkarian ${ }^{1, *}$ and H. A. Stone $\odot^{2, \dagger}$ \\ ${ }^{1}$ Centre de Biochimie Structurale, CNRS UMR 5048-INSERM UMR 1054, University of Montpellier, \\ 34090 Montpellier, France \\ ${ }^{2}$ Department of Mechanical and Aerospace Engineering, Princeton University, Princeton, \\ New Jersey 08544, USA
}

(Received 2 July 2020; accepted 25 August 2020; published 2 October 2020)

\begin{abstract}
Speech is a potent route for viral transmission in the COVID-19 pandemic. Informed mitigation strategies are difficult to develop since no aerosolization mechanism has been visualized yet in the oral cavity. Here we show with high-speed imaging how phonation of common stop consonants, found in most of the world's spoken languages, form and extend salivary filaments in a few milliseconds as moist lips open or when the tongue separates from the teeth. Both saliva viscoelasticity and airflow associated with the plosion of stop consonants are essential for stabilizing and subsequently forming centimeter-scale thin filaments, tens of microns in diameter, that break into speech droplets. Moreover, these plosive consonants induce vortex rings that drive meter-long transport of exhaled air, tying this mechanism to transport associated with speech. We demonstrate that a similar mechanism of aerosolization occurs during the vibration of reeds in wind instruments and may occur during the flapping of the glottis folds. Finally, our research suggests a mitigation of droplet production during speech by using a lip balm.
\end{abstract}

DOI: 10.1103/PhysRevFluids.5.102301

\section{INTRODUCTION}

The COVID-19 pandemic highlights the efficiency of pathogen transmission by salivary and mucus droplets (SMD) [1]. While coughing and sneezing [2-6] are sources of SMD from symptomatic individuals, recent studies document significant transmission of SARS-CoV-2, the virus inducing COVID-19, by asymptomatic and presymptomatic carriers during ordinary conversations [7-11]. Since phonation produces SMD [3,4,12,13] and viral replication occurs mostly in the upper respiratory tract [14], speech might be a potent process for viral transmission. While both SMD size and frequency of production have been measured during speech $[3,4,12,13]$, no aerosolization mechanism has been visualized yet in the oral cavity, which is essential to inform efficient mitigation strategies. In fact, fluid mechanics of saliva and its relationship to airflow in the oral cavity is still in its infancy.

Speaking can be viewed simplistically as the action of strongly modulating exhaled air pressure and flow rate by the rapid vibrations of the glottis folds, together with the movement of the tongue, the lips, and the jaw. The high frequencies of these modulations produce the familiar sounds of speech. Vocalization, as characterized acoustically by linguists [15], can be separated aerodynamically into two classes (using the International Phonetic Alphabet notation /./ for sounds): the

\footnotetext{
*Manouk.Abkarian@umontpellier.fr

†hastone@princeton.edu
} 
production of vowels such as /a/,/ə/,/i/, or /o/, which can be maintained continuously with air exhalation and primarily involves flapping of the glottis folds [16], and the consonants for which the vocal tract is either blocked temporarily or partially. The latter are called obstruent or occlusive consonants and have three major types of articulation: namely, bilabial, formed by movement of the lips, in the case of $/ \mathrm{p} /, / \mathrm{b} /$, or $/ \mathrm{m} /$; alveolar, where the tongue tip is in contact with the alveolar ridge, as for $/ \mathrm{t} /, / \mathrm{d} /$, or $/ \mathrm{n} /$; or velar, using the deeper body of the tongue in contact with the palate as for $/ \mathrm{k} /$ or $/ \mathrm{g} /$. The subsequent pressure buildup and rapid release produce the characteristic burst of airflow of these sounds, which are also called "plosives" [17].

Recently, plosives like /p/ or /b/, which are present in more than $95 \%$ of the world's languages $[18,19]$, have been demonstrated to induce more droplets during speech [13]. Readers may have experienced these emissions, when observing someone's mouth during a conversation in a sufficiently bright spot light. However, both droplet transport and production processes remain uncorrelated and poorly understood. In this work, we focus on such dual mechanisms during speech to identify generic features. All data are experiments performed consistently with one individual (see Supplemental Material Movies S1-S10, and Movie S11 shows similar features with a second individual [20]).

\section{PLOSIVES AND DROPLET PRODUCTION DURING SPEECH}

First, we visualize how specific linguistic features, such as plosive consonants like /p/, are essential for directed transport (see also [21]). In our experiments, a speaker sitting adjacent to a green laser sheet pronounces the syllables "Pa," "Ba," and "Ka," which disturb a mist produced by a fog machine (see Supplemental Material Movie S1 [20]). A high-speed camera (Phantom V7.3, Vision Research) with a 50-mm objective lens, oriented perpendicular to the sheet and operating at 300 frames per second, records the deformation field created by the speaker to obtain by correlation image velocimetry (PIVLab, Matlab) both the velocity and the vorticity fields. For the example of "Pa," we illustrate in Figs. 1(a) and 1(b) the emission of a "puff" with a typical vortex ring structure, which stabilizes the transport of exhaled material over almost a meter. Also, "Ba" presents a vortical structure while "Ka" produces a downward directed jet at almost $50^{\circ}$ from the horizontal (see Supplemental Material Movie S1). In this case, the deeper origin of the vocalization of $/ \mathrm{k} /$, the naturally curved geometry of the oral cavity with the palate, and the subsequent vocalization of /a/, directs the exhaled air jet downward.

Next, to relate the rate of droplet production to the linguistic features of plosives, the speaker stands $15 \mathrm{~cm}$ from the surface of the same laser sheet while pronouncing a series of different consonants associated to the test vowel /a/ at different positions, such as "Pa-aPa-aP" in a repetitive manner. This time, with the high-speed camera at an angle of about $40^{\circ}$, we record at 300 frames per second the region of the sheet in front of the speaker. Emitted droplets are visualized by the scattering they create when crossing the sheet. The distinct spots or flashes allowed us to count the approximate cumulative number of droplets exhaled as a function of time, similar to the method of $[22,23]$; this approach provides a measure of the droplet production rate above the background environmental noise, e.g., dust, and so allows trends to be evaluated at a given place and day. Our first observations show how the pronunciation of a bilabial plosive, such as "Pa," produces a directed cloud of droplets [Fig. 1(c)]. In the case of a denti-alveolar consonant pronounced in "Ta," the droplet count is also large, but the typical angle of emission is directed downward. We repeated the experiments for the different major types of articulation, calmly repeating " $X \mathrm{a}-\mathrm{a} X \mathrm{a}-\mathrm{a} X$," where $X=P, B, K, T, D$, and $F$, over a time period of about 30 s (approximately 10 to 12 repetitions) as displayed in Fig. 1(d). We also compare these cases to voicing ten times the vowels /a/,/ə/,/i/, and /o/. The results show that both bilabial consonants such as $/ \mathrm{p} /$ and $/ \mathrm{b} /$, as well as denti-alveolar plosives $/ \mathrm{t} /$ and $/ \mathrm{d} /$, generate the largest number of droplets per unit time; the numbers are much greater than those for the vowels, consistent with early photographic observations [24]. Surprisingly, the consonant / $\mathrm{k} /$, as in "Ka," had a much lower rate of droplet emission. 
(a)

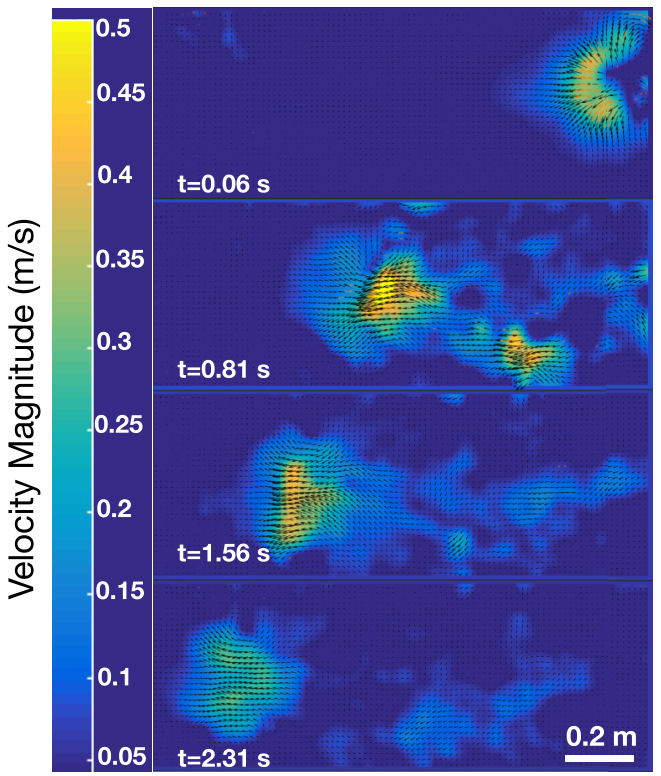

(b)

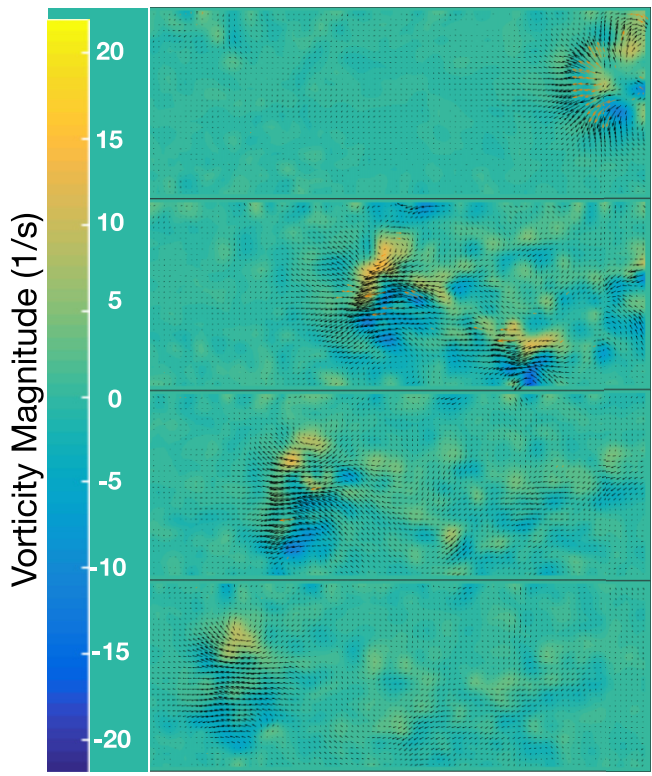

(c)

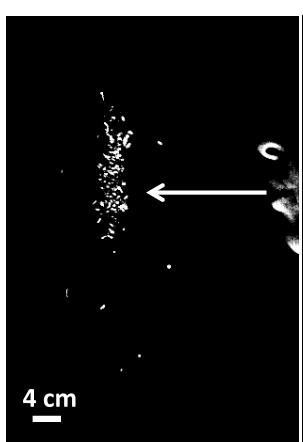

'Pa'

'Ta'

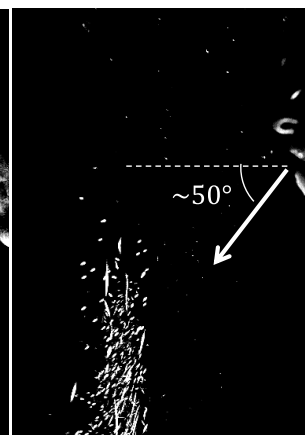

Cumulative Projection over $0.1 \mathrm{~s}$

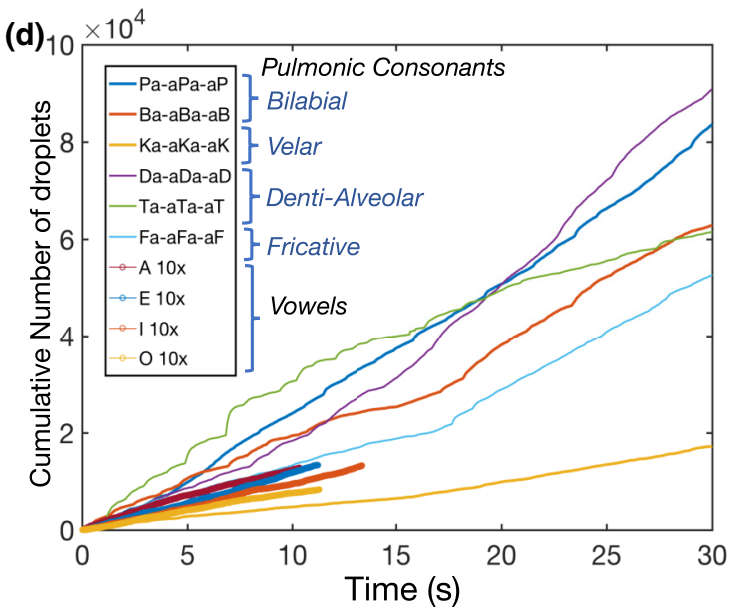

FIG. 1. Plosives, flow, and droplets. (a) Velocity field and (b) vorticity field of the airflow induced by a speaker on the right saying "Pa" adjacent to a laser sheet. (c) Two images of the laser sheet when the speaker says "Pa" or "Ta" directed perpendicular to and at $15 \mathrm{~cm}$ from the sheet. The maximum intensity has been accumulated over $0.1 \mathrm{~s}$ for visualization purposes. (D) Temporal cumulative number of the light flashes representative of the passage of droplets through the laser sheet as a function of time, from repetitively saying " $X \mathrm{a}-\mathrm{a} X \mathrm{a}-\mathrm{a} X$," with $X=/ \mathrm{p} /, / \mathrm{b} /, / \mathrm{k} /, / \mathrm{d} /, / \mathrm{t} /$, and $/ \mathrm{f} /$, with a rapid inspiration in between (approximately 10 to 12 times). We also show the results of saying ten times the vowels $/ \mathrm{a} /, / \mathrm{\partial} /, / \mathrm{i} /, / \mathrm{o} /$.

We conclude that rapid movements in the oral cavity, either of the lips or of the tongue relative to the palate or the teeth, are the source of the high droplet numbers compared to the lower basal level produced when pronouncing only vowels. For the latter, the origin of the droplets might be related to glottis vibrations, which we will discuss later. 


\section{SPEECH, FILAMENT FORMATION, AND BREAKUP}

In a third configuration, we use a high-speed camera, mounted with a bigger 105-mm objective lens (see Materials and Methods, see Ref. [20]), to zoom in on the mouth of the speaker while recording at 5000 frames per second with a strong illumination. The speaker repeats several times the words "PaPa," "BaBa," "TaTa," or "MaMa." The panels of Fig. 2 highlight the syllable "Pa." In panel (a), prior to opening the lips we observe that to maintain sealing and allow the pressurization necessary to pronounce the bilabial consonant "P" in the "Pa" of "PaPa," the lips are pressed together, which spreads a microscopic salivary lubrication layer visible at time $t=0 \mathrm{~ms}$. Then, at the initial stage of detachment of the lips, the liquid layer is put under tension and forms a vertical thin film in a fraction of a millisecond [Fig. 2(a)], which becomes unstable at a distance of about $1 \mathrm{~mm}$ and a timescale of about $1 \mathrm{~ms}$ (Supplemental Material Movie S2); these film thicknesses have an upper bound of about several hundred microns. At $t=2.5 \mathrm{~ms}$, the film has broken up into several filaments that continue to be stretched to approximately $4 \mathrm{~mm}$ by the opening lips. The process is analogous to soft adhesive failure [25], which is more visible if attention is focused on the wedge formed by the separating lips, as seen in Fig. 2(b) (and in Supplemental Material Movie S3). There, the wetting film following this "fracture front" destabilizes continuously into filaments with a separation distance of about $300 \mu \mathrm{m}$. While some filaments remain stable and continue to be stretched, others coalesce with neighboring ones, or break up. When the lips are moistened further by passing several times the tongue over the lips, this failure phenomenon transforms into the receding of a capillary meniscus at an opening wedge (Supplemental Material Movie S4), which ends on the commissure of the lips and seems to act as a natural reservoir of lubricating saliva.

In a second stage, after the first $5 \mathrm{~ms}$, the opening of the lips slows down and a fast airflow bends outward and extends the filaments further by a factor of 3-5 in 5-15 ms. Centimeter-long filaments can be formed as shown in Fig. 2(c) and the time variation of the extension of three filaments is reported in Fig. 2(d), together with the associated separation distance between the lips at the same location. This second phase of fast stretching induces further thinning of the filaments. One example is shown in the inset of Fig. 2(d), where a thick filament with diameter $D_{i} \approx 170 \mu \mathrm{m}$ and an initial length $L_{i} \approx 2.2 \mathrm{~mm}$ forms and thins to $D_{f} \approx 80 \mu \mathrm{m}$ just before snapping off from the lips at a length $L_{f} \approx 10 \mathrm{~mm}$. The ratio $D_{f} / D_{i} \approx 0.47$ is consistent with stretching at constant volume where $L_{i} D_{i}^{2} \approx L_{f} D_{f}^{2}$, leading to $D_{f} / D_{i} \approx \sqrt{L_{i} / L_{f}} \approx \sqrt{0.22} \approx 0.47$. An estimate of the corresponding airflow-induced strain rate gives $\approx 70 \mathrm{~s}^{-1}$. Once a break occurs, the filament whips outward and is stretched further by the airflow, until there is complete detachment and subsequent transport (see Supplemental Material Movie S5); the beads-on-a-string instability [26] is evident, as shown in Fig. 2(e) (see Supplemental Material Movie S6).

Linguistic features such as voicing in bilabial plosive consonants play a role in this mechanism of successive film-to-filament-to-droplet production and transport. Figure 3(a) illustrates the sequence of extension obtained while saying the second "Ba" of "BaBa." While the initial dynamics resemble "PaPa," with salivary spreading in between the pressed lips and vertical film formation at the opening of the lips, the distinct voicing of $/ \mathrm{b} / \mathrm{in}$ "Ba" involves the vibration of the folds of the glottis, while $/ \mathrm{p} /$ is a voiceless consonant [17]. This produces rapid pressure modulations in the airflow that makes the filaments wiggle with a period of $\approx 2-3 \mathrm{~ms}$, as visible on the intensity kymograph of the cross section of one filament in Fig. 3(a) (see Supplemental Material Movie S7). The rapid movements and vibrations destabilize some of the filaments and could be one reason for the lower rate of droplet production for "Ba-aBa-aB" compared to "Pa-aPa-aP" [Fig. 1(d)].

In comparison, the other voiced bilabial consonant $/ \mathrm{m} /$, as in "MaMa," also involves vocal chord vibrations, but since most of the airflow is nasal, the filaments grow and wiggle but break up only over the lips where there is a large opening and very few droplets are ejected from the mouth (Supplemental Material Movie S5). Concomitantly the articulation of denti-alveolar sounds like / $\mathrm{t}$ / in "TaTa" is presented in Fig. 3(c). Here, rapid separation of the tongue from the alveolar ridge and the front teeth induce filament stretching but the larger amount of saliva allows some films to inflate 

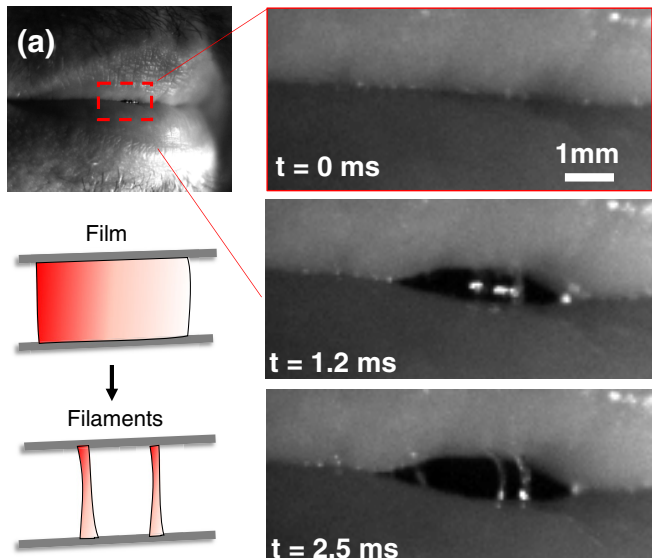
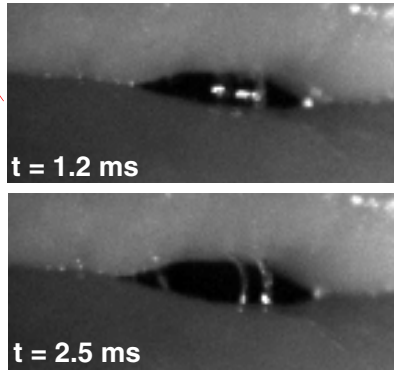

\section{(b)}
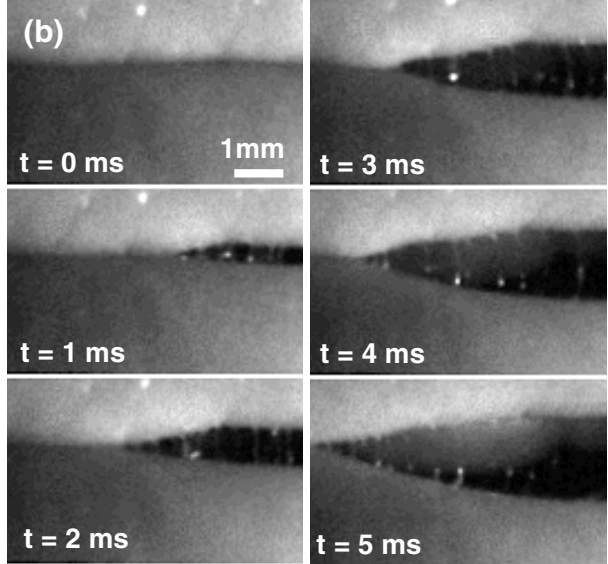

(c)

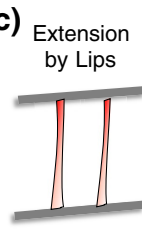

by Air Flow
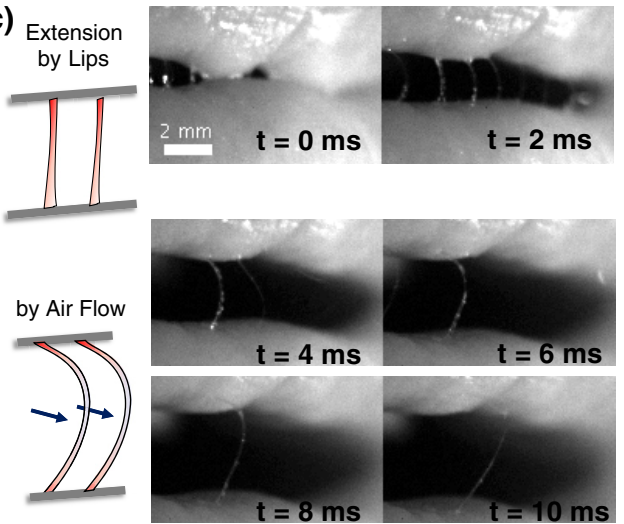

(d)

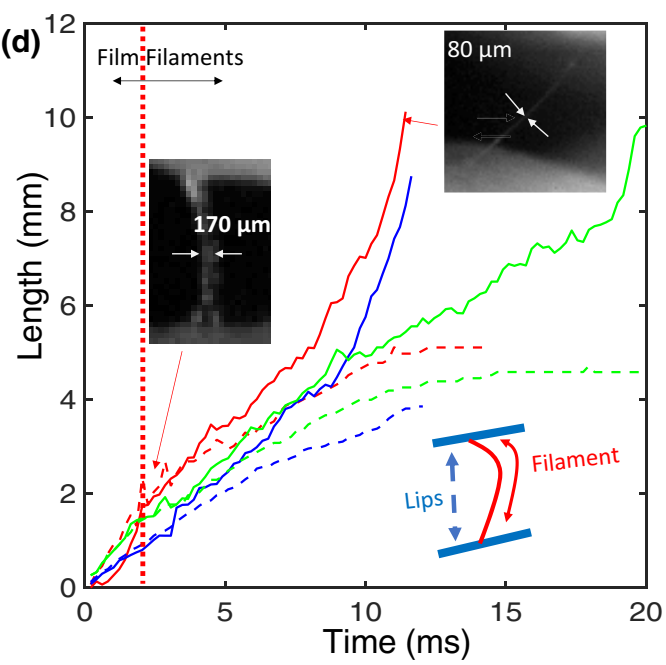

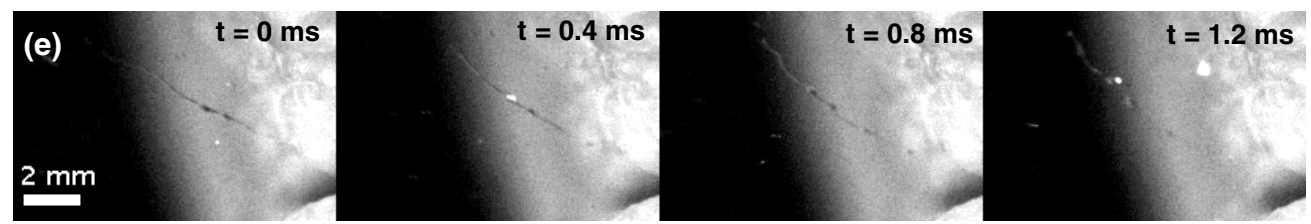

FIG. 2. Film-to-filament-to-droplet. Sequence of images while saying "Pa" in "PaPa" with (a) the initial film formation and destabilization to form filaments, (b) closer view of the junction of the lips as the filaments form, and (c) subsequent sequence of the aerodynamic extension and snapping of the filaments. (d) Measurements of the projected length as a function of time for three filaments (continuous lines) and the associated separation distance between the lips (dashed lines). (e) A flying filament exhibiting the beads-on-a-string instability.

without destabilizing after which they burst rapidly producing a sequence of filaments and droplets (Supplemental Material Movie S6).

The droplet formation clearly involves break-up of liquid filaments. Surface tension $\gamma$ will cause a cylindrical liquid filament to break up into a series of small spheres, which is the Rayleigh-Plateau instability. Both the liquid density, via inertial effects, and viscosity resist the rearrangement of the 
(a)
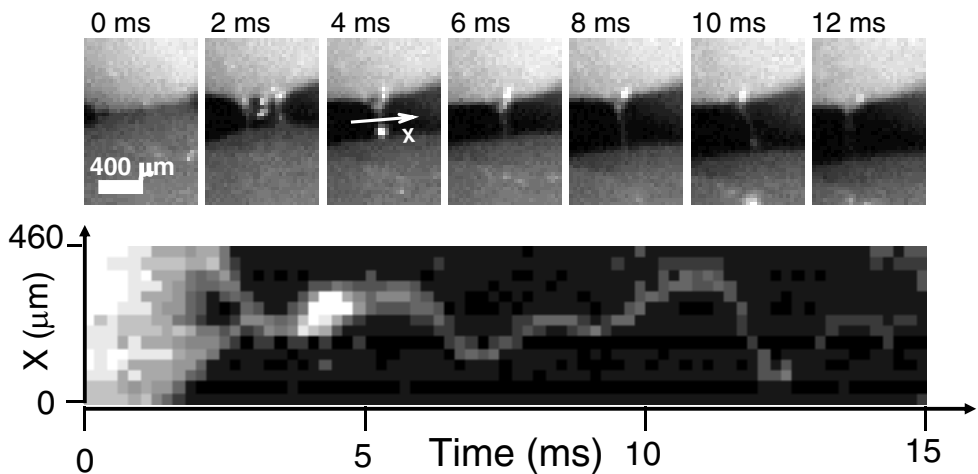

(b)

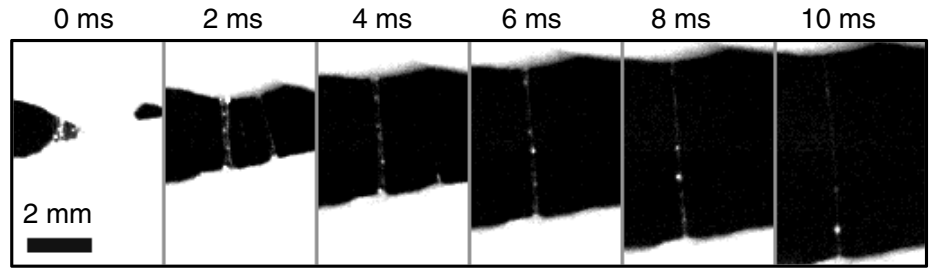

(c)
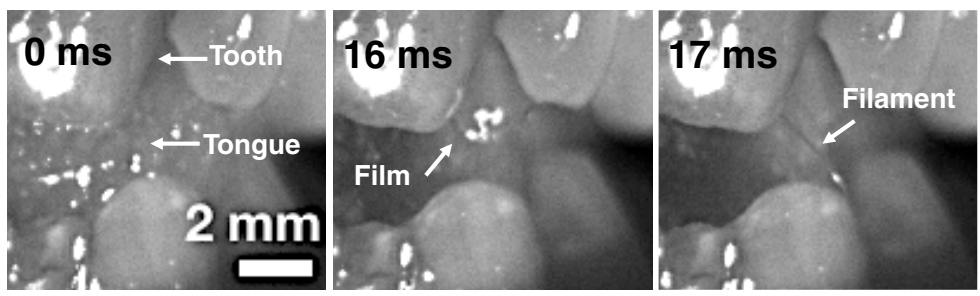

FIG. 3. Filaments and plosive types. (a) Filament stretching and wiggling while saying the second "Ba" in "BaBa" with the associated intensity kymograph. (b) Sequence of one filament stretching while saying the first "Мa" in "MaMa" without visible destabilization from the airflow. (c) Sequence of film-to-filament destabilization between the tongue and the front teeth while saying the second "Ta" in "TaTa."

liquid. Since saliva has an approximate viscosity $\mu \approx 10^{-2} \mathrm{Pas}, \gamma \approx 0.05 \mathrm{~kg} / \mathrm{s}^{2}$, and a density $\rho \approx 10^{3} \mathrm{~kg} / \mathrm{m}^{3}$ comparable to water, then the natural length scale $\mu^{2} /(\rho \gamma) \approx 2 \mu \mathrm{m}$ [27], which is much smaller than the filament radius, $a \approx 40-80 \mu \mathrm{m}$, i.e., the Ohnesorge number $\mathrm{Oh}=\frac{\mu^{2}}{\rho \gamma a} \ll 1$. Hence, inertial effects should be dominant and if the fluid behaved similar to a simple Newtonian liquid, the time for capillary breakup would be approximately $\left(\rho a^{3} / \gamma\right)^{1 / 2} \approx 1 \mathrm{~ms}$.

This estimate shows that a stabilizing material property is present in saliva that permits thinning, leading to droplets of tens of microns in diameter during speech. Several studies have reported saliva relaxation times $\tau_{s}$ of the order of a few to tens of milliseconds [26,28-31]. Since the typical timescale for stretching $\tau_{\text {ext }}$ (lips opening plus airflow) also varies from a few to tens of milliseconds (see Fig. 2), we expect some viscoelastic stabilization since the Deborah number De $=\tau_{s} / \tau_{\text {ext }} \approx 1$, i.e., the large macromolecules in saliva, such as mucin, induce tensile stresses during extensional processes and are unable to relax during articulation.

Moreover, the fundamental frequency of the flapping of vocal folds ranges from $50 \mathrm{~Hz}$ to $>1500 \mathrm{~Hz}$ in singing [17], leading to characteristic opening times from $20 \mathrm{~ms}$ down to $0.6 \mathrm{~ms}$. These timescales suggest that a similar drop formation mechanism is present in the glottis and serves as the origin of the droplets produced during voicing of vowels [see Fig. 2(d)]. We note that some filaments can be observed on images obtained using fast laryngoscopic visualization 

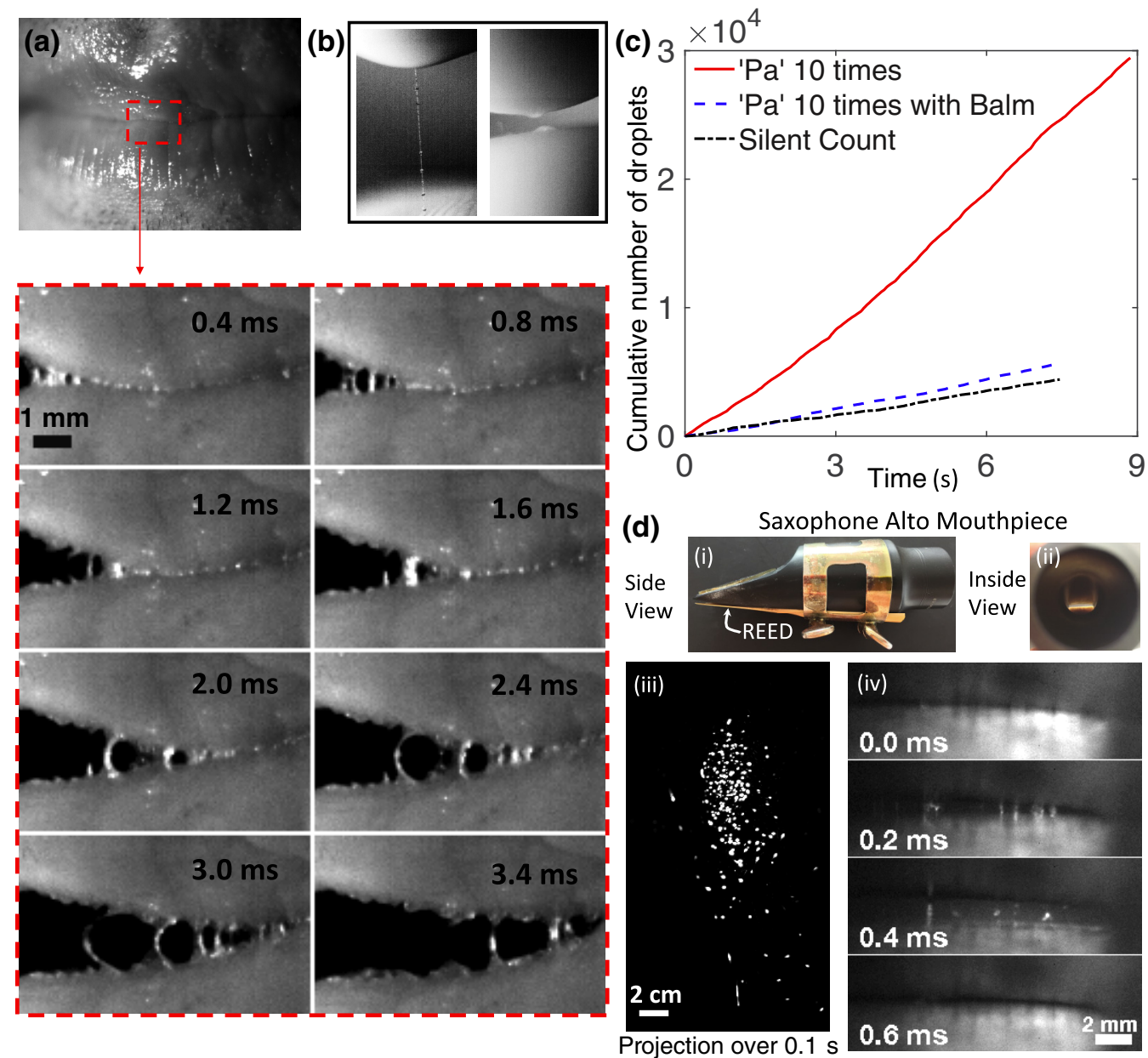

FIG. 4. A lip balm mitigates formation of small droplets. (a) Sequence of images showing the opening of the lips covered with a lip balm while saying the first "Pa" in "PaPa." (b) Saliva droplet spread between two fingers (left) without and (right) with the balm. (c) Temporal average cumulative number of the light flashes representative of the passage of droplets while saying ten times "Pa" over four runs with and without the balm. (d) Droplets production from the mouthpiece (Meyer, Ebonite, Medium Chamber) of a saxophone alto mounted with a wood reed (D’Addario Select Jazz 2H): (i) side view, (ii) inside view, (iii) laser sheet image projected over $0.1 \mathrm{~s}$ when blowing through the mouthpiece constantly, and (iv) high-speed image sequence of filaments and droplets produced between the reed and the tip of the beak, viewed looking towards the beak.

techniques (e.g., see Fig. 4 of [16]). In fact, fast surface separation is the basis of sound production of many woodwind instruments, where the mouthpiece utilizes a vibrating reed, which is wet by saliva and will have similar dynamics. We provide a sequence of images of the dynamics of droplets production in Figs. 4(d)(iii) and 4(d)(iv) where high-speed imaging again demonstrates fast filaments extension, followed by breaking into droplets (see also Supplemental Material Movie S12). Fully understanding the droplet formation requires characterizing the multiple timescales of the problem, e.g., the time to stretch (related to lip opening rate and airflow), the elastic response, the time at which snap off happens, and, finally, the times at which final drop formation occurs.

Over many decades fluid mechanicians have looked at droplets formed in a plethora of situations [32,33] and, more recently, fragmentation of sneeze ejecta have been described [34], where large 
mucus ligaments of several millimeters in diameter lead to large sneeze drops. But the literature of airborne asymptomatic transmission during speech has only indirectly considered two mechanisms [4,6]: (1) bursting of films in the lungs where respiratory passages are blocked during cycles of exhalation and inhalation, which is the so-called bronchial fluid film burst hypothesis [4,35], though, in fact, the authors only measure the final droplet sizes, as do many other researchers $[3,36]$, and (2) theoretical descriptions of shear-induced, airflow-driven instabilities of the mucus fluids lining the respiratory paths [37]. We note that break-up of fluid films due to rapid motion at the glottis or in the mouth have been mentioned, e.g., Johnson and co-workers report an "oral" mode for drop formation [4] without any measurements directly visualizing droplet formation. In contrast, our work shows how saliva viscoelasticity and its wetting, tied to both fast movements of the oral cavity and the plosion of stop consonants, are essential in stabilizing and subsequently stretching saliva filaments over centimeters while thinning them to tens of microns in diameter before breaking into microdroplets.

The relation of the final film thickness, prior to destabilization to form filaments, depends on the initial liquid volume of saliva, its rheology, including possible Marangoni effects [33] and material inhomogeneities, and the stretching rate; this is an interesting and challenging problem for future research. For instance, in the Supplemental Material (see Movie S13) we report a model experiment rapidly pulling a saliva film between two cylindrical plastic tubes, representing the lips: during the stretching, in some cases, we are able to visualize hole formation in the sheets, which is the precursor of filament formation (also seen in the movie), and in each of those cases nucleation occurred at the boundary.

\section{A DROPLET MITIGATION STRATEGY}

Based on these observations and our understanding, it is clear that physicochemical modifications of saliva rheology, relaxation time, or wetting properties over the lips could in principle reduce the stability of filaments or prevent their formation, leading to a possible mitigation of oral aerosolization. We explore one such strategy by likely emulsifying the salivary lubricating film present over the lips while changing its wettability and rheological properties. In Fig. 4, we show preliminary tests using an ordinary lip balm, commonly made of vaseline and shea butter. After applying the balm on the lips [Fig. 4(a)], we realize two types of experiments. First, with high-speed imaging, we demonstrate that indeed filaments break quickly without significant stretching [Fig. 4(a); see Supplemental Material Movie S10]. The mixture of saliva and balm on the lips prevents any large extension of the filaments. Mixing of a saliva droplet in between two fingers covered with balm qualitatively illustrates the change [Fig. 4(b)]. Second, as in Fig. 1(c), we record using a laser sheet the cumulative production rate of droplets upon repeating ten times the syllable "Pa" with and without the balm. We observe a decrease of the cumulative number of droplets produced, reaching almost the ambient noise.

The rheological properties and hydrophobicity of the balm limit the aqueous film formed when passing the tongue over the lips, which is often done by speakers unconsciously when their lips start to dry. In fact, the texture of the balm likely also limits a speaker from moving the tongue over the covered lips. We believe that the film of saliva on the lips is emulsified with the balm when the lips are pressed and separated. Eventually the balm becomes depleted, the lips get rewetted, and drop formation begins again. A future research direction is to develop a balmlike formulation that has a longer lifetime to limit drop formation or act on saliva properties to reduce drop production.

\section{CONCLUSIONS}

In this work we have used high-speed imaging and a scattering-type measurement method for exhaled droplets crossing a laser sheet to provide new insights to the origin of droplets and aerosols during speech. The detailed mechanism involves the viscoelasticity of the saliva and the rapid movements of two wetted surfaces such as the lips, the tongue, and the teeth. The dynamics raise 
the question of the connection of saliva properties to virus transmission, e.g., relaxation times of the saliva have been strongly correlated with an individual's age [28]. Is transmission dependent on this parameter?

Finally, we speculate on superspreaders, who have been suggested to play an outsized role in aerosol transmission of viruses [38,39], including SARS-CoV-2. Superemitters produce an unusually large number of droplets during speech [12] and supershedders have large viral titers in their mucus and saliva and the combination has been suggested to be a defining characteristic of superspreaders. Perhaps both could be linked to their saliva viscoelastic properties and their way of talking? Also, are some languages more prone to produce aerosols? More fundamentally, the connection of droplet production, speech, and saliva rheology raises the question of possible feedback in terms of the evolution of pathogens so as to cause modification of the saliva of their host in order to enhance spreading. Could the saliva of asymptomatic and presymptomatic spreaders, or superspreaders, show modifications induced by the presence of the pathogen that will promote its own aerosolization?

\section{ACKNOWLEDGMENTS}

We thank the NSF for support via the RAPID Grant No. CBET 2029370 (Program Manager is Ron Joslin). We thank the IRN "Physics of Living Systems" (CNRS/INSERM) for support, as well as K. Meersohn for pointing out the importance of plosives in almost all languages of the world and Nan Xue with his help on the laser sheet. We thank Lex Smits for loaning the fog machine. Finally, we thank the MET Orchestra, E. Bowman, S. Levin, and V. Poor for fruitful discussions as part of an ongoing project about musical performances and aerosol production.

[1] L. Bourouiba, Turbulent gas clouds and respiratory pathogen emissions: Potential implications for reducing transmission of COVID-19, J. Am. Med. Assoc. 323, 1837 (2020).

[2] W. F. Wells, On air-borne infection: Study II. Droplets and droplet nuclei, Am. J. Epidemiol. 20, 611 (1934).

[3] J. P. Duguid, The size and the duration of air-carriage of respiratory droplets and droplet-nuclei, Epidemiol. Infect. 44, 471 (1946).

[4] G. Johnson, L. Morawska, Z. Ristovski, M. Hargreaves, K. Mengersen, C. Chao, M.-P. Wan, Y. Li, X. Xie, D. Katoshevski, and S. Corbett, Modality of human expired aerosol size distributions, J. Aerosol Sci. 42, 839 (2011).

[5] L. Bourouiba, E. Dehandschoewercker, and J. W. M. Bush, Violent expiratory events: On coughing and sneezing, J. Fluid Mech. 745, 537 (2014).

[6] R. Mittal, R. Ni, and J.-H. Seo, The flow physics of COVID-19, J. Fluid Mech. 894, F2 (2020).

[7] J. Lu, J. Gu, K. Li, C. Xu, W. Su, Z. Lai, D. Zhou, C. Yu, B. Xu, and Z. Yang, COVID-19 outbreak associated with air conditioning in restaurant, Guangzhou, China, 2020, Emerg. Infect. Dis. 26, 1628 (2020).

[8] S. Y. Park, Y.-M. Kim, S. Lee, B. J. Na et al., Coronavirus disease outbreak in call center, South Korea, Emerging Infect. Dis. 26, 1666 (2020).

[9] L. Hamner, P. Dubbel, I. Capron et al., High SARS-CoV-2 attack rate following exposure at a choir practice-Skagit County, Washington, March 2020, MMWR Morb. Mortal. Wkly. Rep. 69, 606 (2020).

[10] S. Jang, S. H. Han, and J.-Y. Rhee, Coronavirus disease cluster associated with fitness dance classes, South Korea, Emerg. Infect. Dis. 26, 1917 (2020).

[11] D. Hijnen, A. V. Marzano, K. Eyerich, C. GeurtsvanKessel, A. M. Giménez-Arnau, P. Joly, C. Vestergaard, M. Sticherling, and E. Schmidt, SARS-CoV-2 transmission from presymptomatic meeting attendee, Germany, Emerg. Infect. Dis. 26, 1935 (2020).

[12] S. Asadi, A. S. Wexler, C. Cappa, S. Barreda, N. M. Bouvier, and W. D. Ristenpart, Aerosol emission and superemission during human speech increase with voice loudness, Sci. Rep. 9, 2348 (2019). 
[13] S. Asadi, A. S. Wexler, C. Cappa, S. Barreda, N. M. Bouvier, and W. D. Ristenpart, Effect of voicing and articulation manner on aerosol particle emission during human speech, PLoS One 15, e0227699 (2020).

[14] M. Gandhi, D. S. Yokoe, and D. V. Havlir, Asymptomatic transmission, the Achilles' heel of current strategies to control Covid-19, New Engl. J. Med. 382, 2158 (2020).

[15] N. Isshiki and R. Ringel, Air flow during the production of selected consonant, J. Speech Hear. Res. 7, 233 (1964).

[16] C. R. Krausert, A. E. Olszewski, L. N. Taylor, J. S. McMurray, S. H. Dailey, and J. J. Jiang, Mucosal wave measurement and visualization techniques, J. Voice 25, 395 (2011).

[17] C. J. Chen, Elements of Human Voice (World Scientific, Singapore, 2016).

[18] S. J. Styles and L. Gawne, When does maluma/takete fail? Two key failures and a meta-analysis suggest that phonology and phonotactics matter, i-Perception 8, 2041669517724807 (2017).

[19] PHOIBLE 2.0, edited by S. Moran and D. McCloy (Max Planck Institute for the Science of Human History, Jena, 2019).

[20] See Supplemental Material at http://link.aps.org/supplemental/10.1103/PhysRevFluids.5.102301 for Materials and Methods of the experiments as well as text associated with each of the Supplementary Movie files.

[21] M. Abkarian, S. Mendez, N. Xue, F. Yang, and H. A. Stone, Speech can produce jet-like transport relevant to asymptomatic spreading of virus, arXiv:2006.10671 [PNAS (to be published)].

[22] P. Anfinrud, V. Stadnytskyi, C. E. Bax, and A. Bax, Visualizing speech-generated oral fluid droplets with laser light scattering, New Engl. J. Med. 382, 2061 (2020).

[23] D. Grass, I. Henrion, W. S. Warren, E. P. Fischer, M. C. Fischer, and E. Westman, Low-cost measurement of facemask efficacy for filtering expelled droplets during speech, Sci. Adv. 6, eabd3083 (2020).

[24] M. W. Jennison, Atomizing of mouth and nose secretions into the air as revealed by high-speed photography, Aerobiology 17, 106 (1942).

[25] C. Creton and M. Ciccotti, Fracture and adhesion of soft materials: A review, Rep. Prog. Phys. 79, 046601 (2016).

[26] P. P. Bhat, S. Appathurai, M. T. Harris, M. Pasquali, G. H. McKinley, and O. A. Basaran, Formation of beads-on-a-string structures during break-up of viscoelastic filaments, Nat. Phys. 6, 625 (2010).

[27] J. Eggers, Nonlinear dynamics and breakup of free-surface flows, Rev. Mod. Phys. 69, 865 (1997).

[28] E. Zussman, A. L. Yarin, and R. M. Nagler, Age- and flow-dependency of salivary viscoelasticity, J. Dent. Res. 86, 281 (2007).

[29] S. Haward, J. Odell, M. Berry, and T. Hall, Extensional rheology of human saliva, Rheol. Acta 50, 869 (2010).

[30] A. Bazilevsky, V. Entov, and A. Rozhkov, Breakup of a liquid bridge as a method of rheological testing of biological fluids, Fluid Dyn. 46, 613 (2011).

[31] C. E. Wagner and G. H. McKinley, Age-dependent capillary thinning dynamics of physically-associated salivary mucin networks, J. Rheol. 61, 1309 (2017).

[32] E. Villermaux, Fragmentation, Annu. Rev. Fluid Mech. 39, 419 (2007).

[33] E. Villermaux, Fragmentation versus cohesion, J. Fluid Mech. 898, P1 (2020).

[34] B. E. Scharfman, A. H. Techet, J. W. M. Bush, and L. Bourouiba, Visualization of sneeze ejecta: Steps of fluid fragmentation leading to respiratory droplets, Exp. Fluids 57, 24 (2016).

[35] G. R. Johnson and L. Morawska, The mechanism of breath aerosol formation, J. Aerosol Med. Pulm. Drug Delivery 22, 229 (2009).

[36] R. S. Papineni and F. S. Rosenthal, The size distribution of droplets in the exhaled breath of healthy human subjects, J. Aerosol Med. 10, 105 (1997).

[37] J. A. Moriarty and J. B. Grotberg, Flow-induced instabilities of a mucus-serous bilayer, J. Fluid Mech. 397, 1 (1999).

[38] J. O. Lloyd-Smith, S. J. Schreiber, P. E. Kopp, and W. M. Getz, Superspreading and the effect of individual variation on disease emergence, Natur (London) 438, 355 (2005).

[39] P. J. Bueno de Mesquita, C. J. Noakes, and D. K. Milton, Quantitative aerobiologic analysis of an influenza human challenge-transmission trial, Indoor Air (2020). 